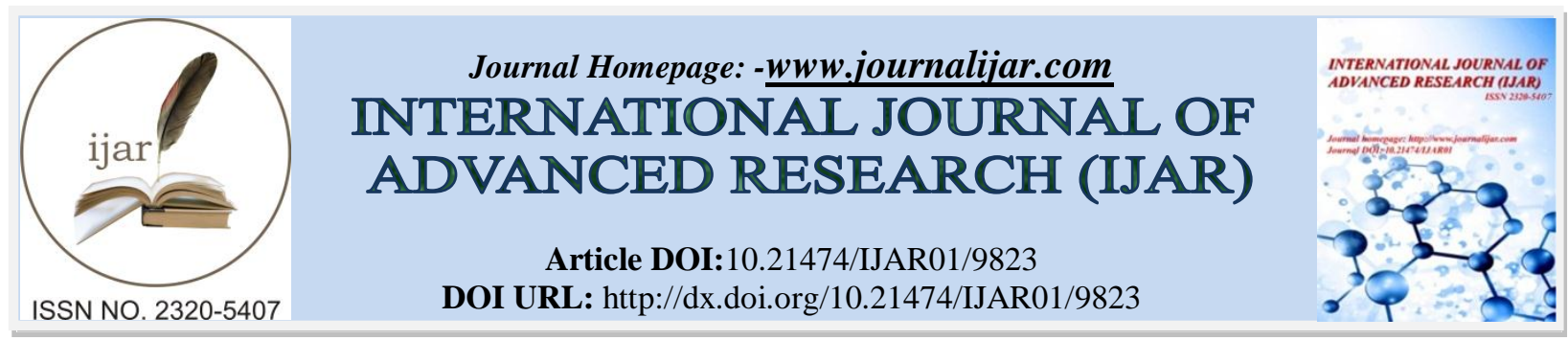

RESEARCH ARTICLE

\title{
CASTLEMAN'S DISEASE: A RARE FINDING IN A PEDIATRIC NECK.
}

\author{
Mohamed Elbouderkaoui, Rida Ait El abdia, Youssef Lakhdar, Youssef Rochdi, Hassan Nouri, Abdelaziz \\ Raji.
}

\section{Manuscript Info}

\section{Manuscript History}

Received: 06 August 2019

Final Accepted: 08 September 2019

Published: October 2019

Key words:-

Castleman, Cervical, Pediatric,

Lymphadenopathy, Surgery.

\begin{abstract}
Castleman's Disease is a rare lymphoproliferative disorder. In the literature, only 29 cases, associated with the neck presentation in children, have been reported. This is another case report regarding a 2year old child who presented with a persistent cervical lymphadenopathy. Final pathology, after undergoing exploratory neck dissection and surgical excision, revealed Castleman's Disease. This report, augmented with a literature review of all the 29 cases, compares the clinical course of this patient with the other cases. In conclusion, although Castleman's Disease carries a favorable prognosis in children, surgical excision is recommended to confirm the diagnosis and to rule out other causes.
\end{abstract}

Copy Right, IJAR, 2019,. All rights reserved.

\section{Introduction:-}

Castleman's Disease (CD), a rare lymphoproliferative disorder [1], was first described in 1954. The disease can occur anywhere in the lymphatic system, but it is most commonly observed in the mediastinum. In fact, only 18 reports (out of 29 pediatric patients) have been found in the literature with the neck involvement $[\mathbf{1}, \mathbf{2}, \mathbf{3}]$. There are two classification systems for CD. The older system, based on the dissemination of the lymph node involvement, divides CD into unicentric and multicentric. Whereas, the new classification system, based on histology, subcategorizes it as hyaline-vascular type, plasma-cell type, and mixed type $[\mathbf{2}, \mathbf{4}]$

\section{Case report}

A 2-year old boy, otherwise healthy, was referred to the Otolaryngology -Head and Neck Surgery service as an outpatient due to a persistent left side neck mass despite multiple courses of antibiotics prescribed by other physicians from different centers. The patient had been suffering from this persistent neck mass for over 1 year. It was slowly progressing but was never tender. He did not have fever, night sweats or any other constitutional symptoms. He had a good appetite and was growing well. He did not get in contact with sick people. His family history was negative for any infectious or oncological disease. During examination of his neck, an approximately 3 $\mathrm{cm}$ firm but not tender right level $\mathrm{V}$ mass was observed. No skin changes over the mass were observed. The mass was fixed and non-fluctuant. The remainder of the physical examination was normal. The blood was normal with a normal range of white blood cell (WBC) and neutrophil counts. CT Scan showed a 32/21 mm homogeneous and hypoechoic lesion in the spinal region (Fig 1). The patient was taken to the operation room for exploratory neck dissection and mass excision. Intraoperatively, the mass was noted to be a large lymph node attached to a chain of small lymph nodes (Fig 2). Complete excision of the clustered lymph nodes was performed. The specimen was sent for pathology that resulted in confirmation of Castleman's Disease. Postoperatively, the patient did well and no complications were faced. No recurrence was observed till 18 months postoperative. The specimen was $2.6 \mathrm{~cm} 3.1$ $\mathrm{cm} 2.2 \mathrm{~cm}$ in size. On cut section, the parenchyma appeared brown in color and homogenous. No necrosis or 
hemorrhage area was identified. Afterwards the specimen was submitted in cassettes for further histological analysis. It showed prominent follicles with regressively transformed germinal centers expanded mangle zone, expanded follicular dendritic networks, hyalinized vessels penetrating germinal centers with prominent interfollicular high endothelial venules. Multiple germinal centers were found in several mangles (Fig 3).Immunohistochemistry did not suggest a neoplastic process. CD20 emphasized both follicles and mangle zones with negative follicle staining for BCL2; CD21 emphasized the expanded follicular dendritic networks; and CD23 emphasized the mantle cell proliferation. CD3 and CD5 stained the prominent interfollicular T-cells. The node was negative for EBER and HHV8; and there was no evidence of monoclonality with kappa and lambda immunostains. CT scann of the chest the abdomen and pelvis was normal. Patient was sent to the hematology for a management supplement. The outcome was positive, no recurrence after one years.

\section{Discussion:-}

There are many causes for persistent cervical lymphadenopathy in the pediatric population, which warrants physicians and surgeons to be diligent on not to misdiagnose a serious disease. After the first description of CD by Benjamin Castleman in 1954 [1]. A literature review by Rabinowitz [2]focusing on paediatric patients with CD in the neck, found 29 patients have been reported in the literature up until 2012.

The patients were aged from 5 to 17 years old with a median age of 12 . Similar to our case they found that $72 \%$ were either asymptomatic or presented with an enlarging neck mass. Hyaline-vascular CD was identified in 28/29 patients, the most common variant in paediatric cases. No recurrence has been reported.[3]. The most common location for a cervical CD is level II/III, which was found in 10 cases, followed by level V in six cases [3]. This case presented with a slow growing mass that was similar to 12/29 cases. This condition occurred below the calculated median age of 12 years of the 29 pediatric cervical CD patients. Additionally, taking into consideration the duration of symptoms, this case is potentially the youngest cervical CD reported so far. The differential diagnosis for a slow growing solitary neck mass is broad. It can include: infection causing lymphadenopathy, branchial cleft cyst, paraganglioma, haemangioma, toxoplasmosis, or more sinister; a lymphoma [4]. The three main imaging modalities to investigate the neck that were used in previously reported cases were Ultrasound, CT Scan, and MRI. Although the most commonly used radiological investigation was a CT Scan (14/29): Castleman disease of the neck can be characterized as solitary or multiple well-defined, mild hypodensity or homogeneous intense lesions on plain CT/MRI scans, and demonstrates intermediate and marked enhancement on contrast-enhanced CT/MRI scans. On double-phase CT scans, Castleman disease often demonstrates mild enhancement at the arterial phase, and gradually uniform enhancement at venous phase. Double-phase enhanced CT or MRI may help to differentiate Castleman disease from other diseases [1-.2-3]. A surgical excision with histopathology and flow cytometry is the gold standard for excluding lymphoma in the setting of an undifferentiatedmass. Castleman's disease does not typically show any monoclonal features on flow cytometry and thus distinguishes it from a lymphoma. There have been case reports of successful treatment with radiotherapy in circumstances where surgical resection isunsafe, these have be shown to reduce the size of the tumour to allow safe surgical excision $[1,5]$.Management of the multicentric disease symptoms can be treated medically with corticosteroids. Biologic agents such as ritixumab and off-label tocilizumab ; this could be considered in a suitable patient with unresectable disease $[4,5]$. Although CD is not a common disease, it shouldbe considered as a possible diagnosis when the patient complains of a neck mass, especially when the patient is suffering from a long-term inflammatorycourse. It is important to remember that all patients confirmed to have CD should receive further evaluation and a systemic survey to exclude the possibility of ignored lesions [5]. To the best of our knowledge, this is one of the young, if not the youngest patient in the literature with cervical CD

\section{Conclusion:-}

Castleman's Disease is a very rare cause of cervical lymphadenopathy in pediatric population. Although all previously reported cases carry a favorable diagnosis, however surgical treatment is recommended to confirm the diagnosis and rule out other more serious conditions.

\section{Conflicts of interest}

The authors declare that there is no conflict of interest in this work. 


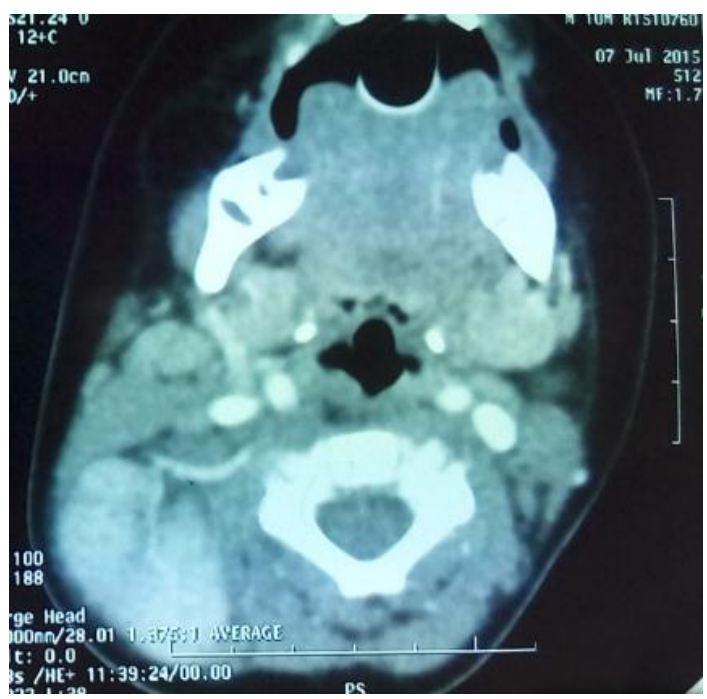

Fig 1:-Contrast-enhanced CT image shows the neck nodules with homogeneous enhancement.

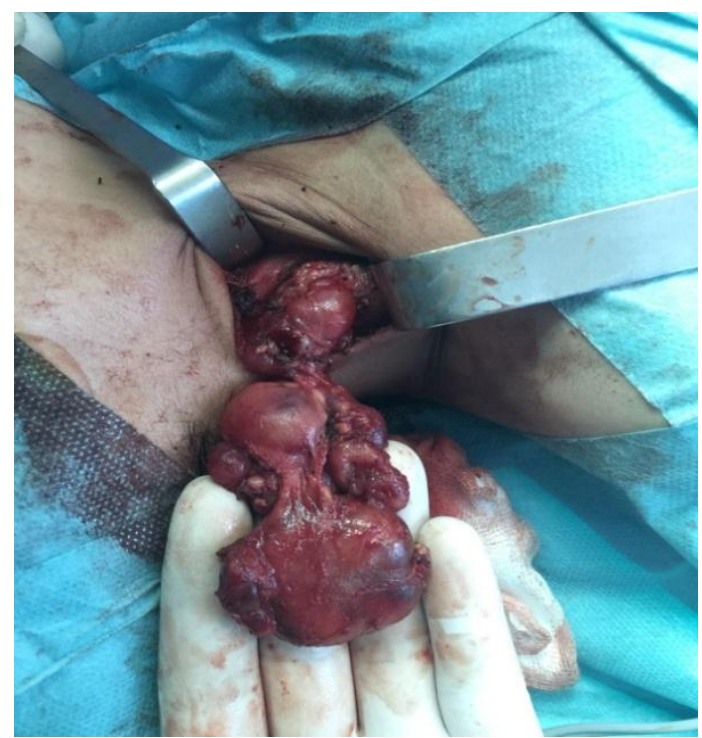

Fig2: Per operative view of the mass.

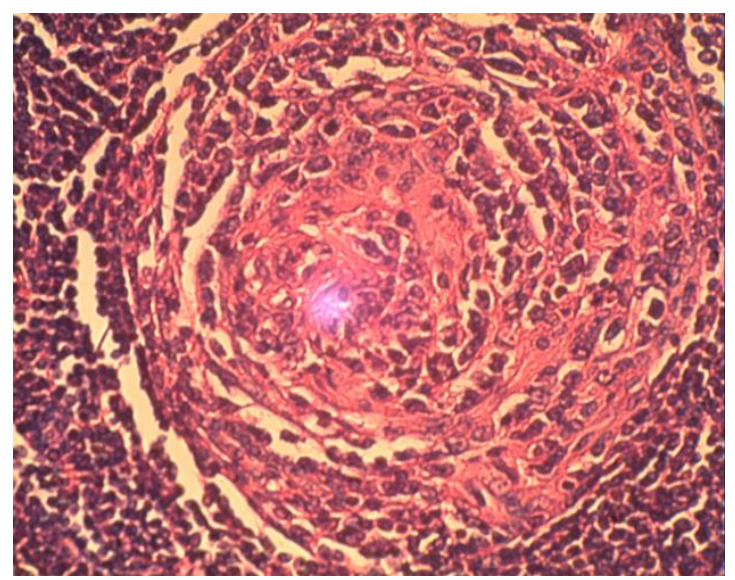

Fig 3:This is a histopathology slide of our patient showing the hyalinized vessels penetrating involuted germinal centers. 


\section{References:-}

1. Faisal Zawawia,c, RickulVarshney a, David G. Haegert b, Sam J. Daniel a, * a Castleman's Disease: A rare finding in a pediatric neck Department of Otolaryngology - Head and Neck Surgery, McGill University, Montreal, QC, Canada b Department of Pathology, McGill University, Montrea

2. M.R. Rabinowitz, J. Levi, K. Conard, U.K. Shah, Castleman disease in the pediatric neck: a literature review, Otolaryngology Head Neck Surg. 148 (2013) 1028- 1036.

3. Hao-ming Song b,1,2, Qing-yu Liuc,3, Yun Cao d,4, Guo-hong Li a,4, Wei-dong Zhang Castleman disease of the neck: CT and MR imaging findings Xin-hua Jianga, 1,4 ,

4. Hannah LinkhornCastleman's disease: An unusually young presentation resulting in delayed diagnosis of a neck mass

5. LinCY ${ }^{1}$, Chang YLCastleman's Disease in the Head and Neck Region: Meta-analysis of Reported Cases in Taiwan and Literature Review..J Formos Med Assoc. 2010 Dec;109(12):913-20. 